\title{
TRATAMENTO QUÍMICO, REVESTIMENTO E ARMAZENAMENTO DE SEMENTES DE Brachiaria brizantha CV. MARANDU ${ }^{1}$
}

\author{
FLÁVIA CARVALHO SANTOS ${ }^{2}$, JOÃO ALMIR OLIVEIRA ${ }^{3}$, ÉDILA VILELA DE RESENDE VON PINHO3, \\ RENATO MENDES GUIMARÃES ${ }^{3}$, ANTÔNIO RODRIGUES VIEIRA ${ }^{3}$
}

\begin{abstract}
RESUMO - A agregação de valor às sementes de Brachiaria spp., utilizando métodos e tecnologias de beneficiamento de sementes como o recobrimento das sementes é uma exigência do mercado consumidor, cada vez mais competitivo. Apesar do incremento no uso de sementes recobertas verificado no mercado nos últimos anos, são poucas as informações disponíveis na literatura sobre o comportamento destas sementes durante o período de armazenamento. Neste contexto, objetivou-se, neste trabalho, avaliar o efeito de diferentes materiais de revestimento em associação com tratamento químico sobre desempenho das sementes durante o armazenamento. Foram utilizadas sementes de Brachiaria brizantha cv. Marandu, e estas foram revestidas em uma betoneira adaptada, utilizando quatorze tipos de revestimento e como testemunha foram utilizadas sementes sem revestimento; combinados com e sem tratamento químico e armazenadas em condição ambiente. As avaliações da qualidade fisiológica pelo teste de germinação e emergência de plântulas e sanitária foram realizadas durante 0, 3, 6, 9 e 12 meses de armazenamento. Conclui-se que sementes revestidas com Areia + PVA e Areia $+\mathrm{CaSiO}_{3}+$ PVA ou polímero, tratadas quimicamente, mantém sua qualidade fisiológica ao longo dos 12 meses de armazenamento; o tratamento químico com fungicida Carbendazim + Thiram e inseticida Fipronil nas sementes revestidas ou não, é eficiente no controle de fungos e promove melhor desempenho fisiológico ao longo do armazenamento; o revestimento com Betonita e/ou Calcário afeta negativamente a qualidade fisiológica das sementes de Brachiaria brizantha cv. Marandu no armazenamento por um período de 12 meses; o revestimento de sementes promove redução da velocidade de germinação e emergência de sementes de Brachiaria brizantha cv. Marandu.
\end{abstract}

Termos para indexação: qualidade fisiológica; recobrimento de sementes; forrageira.

\section{CHEMICAL TREATMENT, SEED COATING AND STORAGE OF Brachiaria brizantha CV. MARANDU SEEDS}

\begin{abstract}
Despite the increasing use of coated seeds in recent years, there is little information available on the behavior of these seeds during storage. Therefore, the aim of this study was to evaluate the effect of different coating materials, in combination with chemical treatments, on seed performance during storage. Seeds of Brachiaria brizantha cv. Marandu provided by WolfSeeds were coated (pelleted) in an adapted concrete mixer using fourteen types of coating and were compared to uncoated control seeds. The combination of these treatments was also evaluated with fungicide and insecticide treatments of the seed. An evaluation was made of the physiological quality from the germination test, seedling emergence and sanitary quality after $0,3,6,9$ and 12 months of storage. It was concluded that seeds coated with Sand $+\mathrm{PVA}$ and sand $+\mathrm{CaSiO}_{3}+\mathrm{PVA}$ or polymer, chemically
\end{abstract}

\footnotetext{
${ }^{1}$ Submetido em 14/09/2009. Aceito para publicação em 20/04/2010. Parte da Tese de Doutorado em Agronomia/Fitotecnia, pelo primeiro autor.

${ }^{2}$ Eng. Agr. Doutora pela Universidade Federal de Lavras, Caixa Postal 37,
}

CEP: 37200-000, Lavras - MG, flavinha.agronomia@gmail.com.

${ }^{3}$ Professor Dr., Universidade Federal de Lavras, Caixa Postal 37, CEP: 37200-000, Lavras - MG, jalmir@dag.ufla.br 
treated, maintained physiological quality over the 12 months of storage; chemical treatment with the fungicide mixture Carbendazim + Thiram and the insecticide Fipronil on coated or non-coated seed gives effective control of fungi and promotes better physiological performance during storage; the coating with bentonite and/ or limestone negatively affected the seed quality of Brachiaria brizantha cv. Marandu during the 12 months storage period; the seed coating reduced the speed of germination and emergence of Brachiaria brizantha cv. Marandu.

Index termis: physiological quality; coated seed; forage.

\section{INTRODUÇÃO}

O Brasil é o maior produtor, consumidor e exportador mundial de sementes de Brachiaria $s p$. Nas duas últimas décadas tem sido notável o aumento da área de pastagens formadas com gramíneas do gênero Brachiaria, acompanhado de um aumento proporcional na produção e comercialização de suas sementes (Martins et al., 1998). Diversos agentes interagem nesse processo de produção, que representa um faturamento anual de milhões de dólares e a manutenção de milhares de empregos. Apesar da grande importância, o uso de sementes de má qualidade é causa frequente de fracasso na formação de áreas de pastagens e os investimentos em pesquisa são escassos.

A agregação de valor às sementes de Brachiaria spp., utilizando métodos e tecnologias de beneficiamento de sementes como a do revestimento, é uma exigência de um mercado cada vez mais competitivo.

$\mathrm{O}$ revestimento de sementes consiste na deposição de um material seco, inerte e um material cimentante (adesivo) à superfície da semente, permitindo a modificação ou não da forma e tamanho da semente (Silva et al., 2002).

A utilização de sementes revestidas possibilita a redução dos custos de produção de mudas, diminuindo o consumo de sementes, facilita a mecanização da semeadura, além de proporcionar melhorias na sanidade das sementes e no estabelecimento das plântulas através da incorporação de nutrientes, reguladores de crescimento e outros agroquímicos (Silva et al., 2002; Bonome, 2003).

$\mathrm{O}$ uso de fungicidas para tratamento químico de sementes é um dos métodos de mais baixo custo no controle integrado de doenças de plantas, não só com o interesse de eliminar os patógenos associados às sementes, mas também proteger as sementes e plântulas, durante sua fase inicial de desenvolvimento, de agentes patogênicos presentes na semente e no solo (Goulart, 2000).

O processo de produção de sementes é constituído de várias etapas e uma delas, não menos importante que as demais, é a do armazenamento. A preservação da qualidade das sementes durante o armazenamento é um aspecto fundamental a ser considerado no processo produtivo, pois os esforços despendidos na fase de produção podem ser infrutíferos se a qualidade das sementes não for mantida, no mínimo até a época de semeadura (Oliveira et al., 1999). Dentre as medidas preventivas, a aplicação de fungicidas e inseticidas visando à proteção de sementes durante o armazenamento torna-se cada dia mais importante na produção agrícola.

Apesar do incremento no uso de sementes recobertas verificado no mercado nos últimos anos, são poucas as informações disponíveis na literatura sobre o comportamento destas sementes durante o armazenamento.

Desta forma, torna-se necessário que os materiais utilizados no revestimento das sementes sejam estudados juntamente com o tratamento químico, já que alguns deles podem causar efeitos fitotóxicos imediatos na germinação ou reduzir a qualidade fisiológica das sementes.

Portanto, objetivou-se, neste trabalho, avaliar o efeito do revestimento em associação com tratamento fungicida e inseticida sobre desempenho das sementes durante o armazenamento, em condições de ambiente.

\section{MATERIAL E MÉTODOS}

As sementes utilizadas no experimento foram armazenadas na unidade de beneficiamento de sementes (UBS) e analisadas no Laboratório Central de Sementes do Departamento de Agricultura e Setor de Patologia de Sementes do Departamento de Fitopatologia da Universidade Federal de Lavras (UFLA).

Foram utilizadas sementes sem escarificação de Brachiaria brizantha cv. Marandu, fornecidas pela WolfSeeds. O lote de sementes foi revestido (peletizados) em uma betoneira adaptada, utilizando quatorze tipos de revestimento: areia + PVA; areia + polímero Lanxess $\AA$; calcário + PVA; calcário + polímero Lanxess ${ }^{\circledR}$; areia + 
betonita $(3: 1)+$ PVA; areia + betonita $(3: 1)+$ polímero Lanxess ${ }^{\circledR}$; calcário + betonita $(3: 1)+$ PVA; calcário + betonita $(3: 1)+$ polímero Lanxess ${ }^{\circledR}$; areia + calcário $(2: 1)$ + PVA; areia + calcário $(2: 1)+$ polímero Lanxess ${ }^{\circledR}$; areia + silicato de cálcio (3:1) + PVA; areia + silicato de cálcio $(3: 1)+$ polímero Lanxess ${ }^{\circledR}$; calcário + silicato de cálcio (3:1) + PVA; calcário + silicato de cálcio $(3: 1)+$ polímero Lanxess ${ }^{\circledR}$ e ainda sementes sem revestimento; combinados ou não com fungicida e inseticida. $\mathrm{O}$ tratamento químico consistiu na mistura de Carbendazim + Thiram $(150+350)$ SC (dosagem $200 \mathrm{~mL} / 100 \mathrm{~kg}$ sementes) e Fipronil (40 $\mathrm{mL} / 100 \mathrm{~kg}$ sementes) para posterior revestimento. $\mathrm{O}$ adesivo PVA (acetato de polivinil) foi utilizado na concentração de $20 \%(\mathrm{v} / \mathrm{v})$.

Foi criado para execução dos experimentos, um protótipo do equipamento de peletização. Cada ingrediente cimentante foi aplicado por pulverização dirigida à massa circulante dentro da betoneira, recebendo aos poucos os ingredientes de enchimento e agente cimentante (PVA ou o polímero) até que estes aderissem à superfície da semente em camadas sucessivas até atingir o tamanho desejado.

Frequentemente os péletes foram peneirados, utilizando peneira Bertel ${ }^{\circledR} 6$ (abertura $3,35 \mathrm{~mm}$ ); os péletes que ficavam retidos na peneira eram separados e os restantes retornavam à betoneira para dar continuidade ao processo. Após todos os péletes adquirirem o tamanho desejado, os mesmos foram secados em estufa de circulação forçada de ar, a $35^{\circ} \mathrm{C}$, por 24 horas.

Posteriormente, as sementes foram acondicionadas em embalagens de papel multifoliado e armazenadas em condição ambiente. A qualidade fisiológica e sanitária das sementes foram avaliadas aos 0, 3, 6, 9 e 12 meses pelas seguintes avaliações:

Teste de germinação: A semeadura foi realizada em caixas plásticas transparentes (tipo gerbox 11,5 x 11,5 x 3,5 $\mathrm{cm})$, com 4 repetições de 50 sementes, distribuídas sobre duas folhas de papel mata-borrão, umedecidas com água em quantidade equivalente a 2,5 vezes o peso do mesmo. A seguir, as caixas de gerbox foram transferidas para a câmara de germinação (BOD), em regime alternado de luz e de escuro (12 horas), regulada à temperatura alternada de 20 e $30^{\circ} \mathrm{C}$. As avaliações foram feitas aos 7 e 21 dias, computando-se o número de plântulas normais, de acordo com os critérios estabelecidos nas Regras para Análise de Sementes (Brasil, 1992), sendo os resultados expressos em porcentagem.

Teste de emergência de plântulas: A semeadura foi realizada em substrato solo + areia na proporção 1:2 em bandejas plásticas. A umidade do substrato foi ajustada para $60 \%$ da capacidade de retenção. Foram utilizadas 4 repetições de 50 sementes por tratamento. A profundidade da semeadura foi de $1 \mathrm{~cm}$ e as bandejas mantidas em câmara de crescimento vegetal, previamente regulada à temperatura de $25{ }^{\circ} \mathrm{C}$ em regime alternado de luz e escuro (12 horas). As bandejas foram irrigadas quando necessário. A partir da emergência, foram realizadas avaliações diárias, computando-se o número de plântulas emergidas, até a estabilização. O índice de velocidade de emergência foi calculado segundo fórmula proposta por Maguirre (1962). A porcentagem de emergência das plântulas foi avaliada após a estabilização da emergência nas parcelas, verificando-se o número de plântulas normais emergidas.

O experimento foi constituído de um esquema fatorial $15 \times 2 \times 5$ (15 tipos de revestimento, com e sem tratamento químico e 5 períodos de armazenamento de 0, 3, 6, 9 e 12 meses), em delineamento inteiramente casualizado. Os dados foram analisados utilizando-se o pacote computacional SISVAR, versão 4.0 (Ferreira, 2000). As médias dos fatores épocas de armazenamento foram comparadas por meio do teste de regressão e as médias dos fatores tratamento químico e do fator revestimentos pelo Teste de Scott-Knott.

\section{RESULTADOS E DISCUSSÃO}

Verificou-se efeito significativo da interação tripla em todas as variáveis analisadas, indicando que os três fatores (revestimento, tratamento químico e armazenamento) estão interagindo ou são dependentes, com um dos fatores influenciando na ação dos outros dois.

$\mathrm{Na}$ Tabela 1 estão apresentadas as médias dos resultados do teste de germinação. Verifica-se que as sementes recémarmazenadas (zero mês de armazenamento), tratadas quimicamente, e revestidas com Areia + PVA e Areia + polímeroresultaramemmaioresporcentagens degerminação, não diferindo das sementes sem revestimento e, menores porcentagens foram obtidas com sementes revestidas com Calcário + Betonita + PVA e Calcário $+\mathrm{CaSiO}_{3}+$ polímero. Aos três meses de armazenamento as sementes revestidas com Areia $+\mathrm{PVA}$, Areia $+\mathrm{CaSiO}_{3}+\mathrm{PVA}$, Areia $+\mathrm{CaSiO}_{3}+$ polímero resultaram em maiores porcentagens de germinação, não apresentando diferenças significativas em relação à testemunha, e as menores porcentagens foram constatados nas revestidas com Calcário $+\mathrm{CaSiO}_{3}$ + polímero. Aos seis meses de armazenamento melhores resultados foram obtidos por meio das sementes revestidas com Areia + PVA, Areia $+\mathrm{CaSiO}_{3}+$ PVA, não diferindo 
estatisticamente da testemunha, e o menor resultado foi observado utilizando sementes revestidas com Calcário + Betonita + polímero. Para as sementes com nove meses de armazenamento, pôde-se verificar que as revestidas com Areia + PVA, Areia + polímero, Areia + Betonita +
PVA, Areia $+\mathrm{CaSiO}_{3}+$ PVA, Areia $+\mathrm{CaSiO}_{3}+$ polímero, propiciaram maiores valores de germinação, não diferindo significativamente da testemunha (sem revestimento), e nas revestidas com Calcário + Betonita + polímero, Calcário + Betonita + PVA foram observados os menores valores.

TABELA 1. Resultados médios (\%) do teste de germinação de sementes de Brachiaria brizantha cv. Marandu revestidas com diferentes materiais ( $\mathrm{A}$ - areia, $\mathrm{C}$ - calcário, $\mathrm{AB}$ - areia + betonita, $\mathrm{CB}$ - calcário + betonita, $\mathrm{ACaSiO}_{3}$ - areia $+\mathrm{CaSiO}_{3}, \mathrm{CCaSiO}_{3}-$ calcário $+\mathrm{CaSiO}_{3}$ ), submetidas ao tratamento químico (TQ) ou não aos 0, 3, 6, 9 e 12 meses de armazenamento.

\begin{tabular}{|c|c|c|c|c|c|c|c|c|c|c|}
\hline \multirow{3}{*}{ Revestimento } & \multicolumn{10}{|c|}{ Armazenamento (meses) } \\
\hline & \multicolumn{2}{|c|}{0} & \multicolumn{2}{|c|}{3} & \multicolumn{2}{|c|}{6} & \multicolumn{2}{|c|}{9} & \multicolumn{2}{|c|}{12} \\
\hline & $\begin{array}{c}\text { Com } \\
\text { TQ }\end{array}$ & $\begin{array}{c}\text { Sem } \\
\text { TQ }\end{array}$ & $\begin{array}{c}\text { Com } \\
\text { TQ }\end{array}$ & $\begin{array}{c}\text { Sem } \\
\text { TQ }\end{array}$ & $\begin{array}{c}\text { Com } \\
\text { TQ }\end{array}$ & $\begin{array}{c}\text { Sem } \\
\text { TQ }\end{array}$ & $\begin{array}{c}\text { Com } \\
\text { TQ }\end{array}$ & $\begin{array}{c}\text { Sem } \\
\text { TQ }\end{array}$ & $\begin{array}{c}\text { Com } \\
\text { TQ }\end{array}$ & $\begin{array}{c}\text { Sem } \\
\text { TQ }\end{array}$ \\
\hline $\mathrm{A}+\mathrm{PVA}$ & $64 \mathrm{Aa}$ & $57 \mathrm{Ba}$ & $78 \mathrm{Aa}$ & $69 \mathrm{Cb}$ & $76 \mathrm{Aa}$ & $64 \mathrm{Ca}$ & $71 \mathrm{Aa}$ & $62 \mathrm{Ca}$ & $67 \mathrm{Aa}$ & $58 \mathrm{Ba}$ \\
\hline A + pol. & $67 \mathrm{Aa}$ & $51 \mathrm{Cb}$ & $68 \mathrm{Ba}$ & $65 \mathrm{Ca}$ & $73 \mathrm{Ba}$ & $61 \mathrm{Cb}$ & $72 \mathrm{Aa}$ & $57 \mathrm{Db}$ & 64Aa & $55 \mathrm{Bb}$ \\
\hline $\mathrm{C}+\mathrm{PVA}$ & $43 \mathrm{Ca}$ & $40 \mathrm{Ea}$ & $63 \mathrm{Ba}$ & $50 \mathrm{Db}$ & $54 \mathrm{Ea}$ & $47 \mathrm{Db}$ & $51 \mathrm{Ca}$ & $44 \mathrm{~Eb}$ & $45 \mathrm{Da}$ & $41 \mathrm{Ca}$ \\
\hline $\mathrm{C}+$ pol. & $37 \mathrm{Ea}$ & $23 \mathrm{Hb}$ & $54 \mathrm{Ca}$ & $32 \mathrm{Fb}$ & $66 \mathrm{Ca}$ & $38 \mathrm{~Eb}$ & $50 \mathrm{Ca}$ & $34 \mathrm{Fb}$ & 43Da & $33 \mathrm{Db}$ \\
\hline $\mathrm{AB}+\mathrm{PVA}$ & 39Da & $25 \mathrm{Hb}$ & $68 \mathrm{Ba}$ & $30 \mathrm{Fb}$ & $70 \mathrm{Ba}$ & $61 \mathrm{Cb}$ & 66Aa & $53 \mathrm{Db}$ & $58 \mathrm{Ba}$ & $39 \mathrm{Cb}$ \\
\hline $\mathrm{AB}+$ pol. & $55 \mathrm{Ba}$ & $22 \mathrm{Hb}$ & $61 \mathrm{Ba}$ & $26 \mathrm{Fb}$ & $61 \mathrm{Da}$ & $38 \mathrm{~Eb}$ & $56 \mathrm{Ba}$ & $34 \mathrm{Fb}$ & $51 \mathrm{Ca}$ & $30 \mathrm{Db}$ \\
\hline $\mathrm{CB}+\mathrm{PVA}$ & $33 \mathrm{Fa}$ & $23 \mathrm{Hb}$ & 47Da & $52 \mathrm{Da}$ & $54 \mathrm{~Eb}$ & $70 \mathrm{Ba}$ & $45 \mathrm{Db}$ & $60 \mathrm{Ca}$ & $41 \mathrm{Db}$ & $47 \mathrm{Ca}$ \\
\hline $\mathrm{CB}+$ pol. & $46 \mathrm{Ca}$ & $42 \mathrm{Ea}$ & $47 \mathrm{Da}$ & $44 \mathrm{Ea}$ & $45 \mathrm{Fa}$ & $42 \mathrm{Ea}$ & $43 \mathrm{Da}$ & $42 \mathrm{Ea}$ & $41 \mathrm{Da}$ & $43 \mathrm{Ca}$ \\
\hline $\mathrm{AC}+\mathrm{PVA}$ & $40 \mathrm{Da}$ & $36 \mathrm{Fb}$ & $66 \mathrm{Ba}$ & $55 \mathrm{Db}$ & $55 \mathrm{Ea}$ & $50 \mathrm{Db}$ & $49 \mathrm{Ca}$ & $45 \mathrm{~Eb}$ & $42 \mathrm{Da}$ & $43 \mathrm{Ca}$ \\
\hline $\mathrm{AC}+$ pol. & $45 \mathrm{Ca}$ & $29 \mathrm{~Gb}$ & $66 \mathrm{Ba}$ & $55 \mathrm{Db}$ & $68 \mathrm{Ca}$ & $43 \mathrm{~Eb}$ & $59 \mathrm{Ba}$ & $39 \mathrm{Fb}$ & $51 \mathrm{Ca}$ & $32 \mathrm{Db}$ \\
\hline $\mathrm{ACaSiO}_{3}+\mathrm{PVA}$ & $56 \mathrm{Ba}$ & $45 \mathrm{Db}$ & $74 \mathrm{Aa}$ & $76 \mathrm{Ba}$ & $80 \mathrm{Aa}$ & 82Aa & $73 \mathrm{Aa}$ & $74 \mathrm{Aa}$ & $70 \mathrm{Aa}$ & $67 \mathrm{Aa}$ \\
\hline $\mathrm{ACaSiO}_{3}+$ pol. & $42 \mathrm{Db}$ & $53 \mathrm{Ca}$ & $72 \mathrm{Aa}$ & $74 \mathrm{Ba}$ & $71 \mathrm{Ba}$ & 76Aa & $72 \mathrm{Aa}$ & $67 \mathrm{Bb}$ & $68 \mathrm{Aa}$ & $61 \mathrm{Bb}$ \\
\hline $\mathrm{CCaSiO}_{3}+\mathrm{PVA}$ & $54 \mathrm{Ba}$ & $58 \mathrm{Ba}$ & $66 \mathrm{Ba}$ & $63 \mathrm{Ca}$ & $65 \mathrm{Ca}$ & $66 \mathrm{Ca}$ & $61 \mathrm{Ba}$ & $63 \mathrm{Ca}$ & $55 \mathrm{Ca}$ & $58 \mathrm{Ba}$ \\
\hline $\mathrm{CCaSiO}_{3}+$ pol. & $31 \mathrm{Fa}$ & $32 \mathrm{Fa}$ & $39 \mathrm{~Eb}$ & $46 \mathrm{Ea}$ & $53 \mathrm{Ea}$ & $52 \mathrm{Da}$ & $59 \mathrm{Ba}$ & $46 \mathrm{~Eb}$ & $53 \mathrm{Ca}$ & $44 \mathrm{Cb}$ \\
\hline S/ revestimento & $70 \mathrm{Aa}$ & $66 \mathrm{Aa}$ & 77Aa & 80Aa & $78 \mathrm{Aa}$ & $78 \mathrm{Aa}$ & $74 \mathrm{Aa}$ & $70 \mathrm{Ba}$ & $72 \mathrm{Aa}$ & 69Aa \\
\hline
\end{tabular}

Médias seguidas pela mesma letra maiúscula na coluna e minúscula na linha para cada tipo de escarificação não diferem estatisticamente entre si pelo Teste de Scott-Knott, a 5\% de probabilidade.

Observa-se, ainda nas sementes tratadas quimicamente, que aos 12 meses, Areia + PVA, Areia + polímero, Areia $+\mathrm{CaSiO}_{3}+\mathrm{PVA}$, Areia $+\mathrm{CaSiO}_{3}+$ polímero foram os revestimentos que resultaram em maiores porcentagens de germinação, sem haver diferença significativa em relação às sem revestimento. As sementes revestidas com Calcário + PVA, Calcário + polímero, Calcário + Betonita + PVA, Calcário + Betonita + polímero, Areia + Calcário + PVA resultaram em menores porcentagens de germinação.

Além das sementes não revestidas, nas revestidas com Areia + PVA e tratadas quimicamente observou-se maior porcentagem de germinação em todas as épocas de armazenamento. Pereira et al. (2005) observaram que as sementes revestidas com areia apresentaram um ótimo desempenho antes do armazenamento, no entanto sua qualidade foi reduzida linearmente ao longo do armazenamento.

Todavia, quando se realizaram avaliações nas sementes revestidas, não tratadas durante $0,3,6,9$ e 12 meses de armazenamento (Tabela 1) pode-se verificar que, nas sementes recém-armazenadas (zero meses de armazenamento) e aos três meses de armazenamento, nas sem revestimento, foi observada maior porcentagem de germinação que aquelas revestidas com os diferentes materiais. Menor porcentagem foi obtida utilizando sementes revestidas com Calcário + polímero, Areia + 
Betonita + PVA, Areia + Betonita + polímero, Calcário + Betonita + PVA, onde a menor porcentagem nos três meses só não foi apresentada naquelas revestidas com Calcário + Betonita + PVA, já os demais tratamentos apresentaram o mesmo valor que as sementes recém-armazenadas. Nas sementes armazenadas com seis meses, maiores valores de germinação foram observados nas revestidas com Areia + $\mathrm{CaSiO}_{3}+\mathrm{PVA}$, Areia $+\mathrm{CaSiO}_{3}+$ polímero, não apresentando diferença em relação à testemunha. Com nove meses de armazenamento as sementes revestidas com Areia $+\mathrm{CaSiO}_{3}$ + PVA resultou em maior porcentagem de germinação superando a testemunha e menor porcentagem foi observada nas revestidas com Calcário + polímero, Areia + Betonita + polímero e Areia + Calcário + polímero.

Ainda nas sementes não tratadas, observou-se aos 12 meses de armazenamento, maior porcentagem de germinação naquelas revestidas com Areia $+\mathrm{CaSiO}_{3}+\mathrm{PVA}$, em relação às demais, não diferindo da testemunha, e Calcário + polímero, Areia + Betonita + polímero e Areia + Calcário + polímero menores valores. Provavelmente, o fato das sementes revestidas contendo Areia $+\mathrm{CaSiO}_{3}+$ PVA apresentarem melhores resultados que os demais ao longo do armazenamento possa ser atribuído ao efeito de silício no controle de patógenos.

Observou-se que, sementes revestidas com areia tiveram maior facilidade para protrusão radicular que as sementes revestidas com Areia + Betonita. A betonita é um material higroscópico, a qual em contato com a água fica mais tempo úmida, em relação aos demais materiais de revestimento, e além dessa higroscopicidade, ela é um material compacto, fazendo com que as sementes revestidas com esse material tenham dificuldade de absorver oxigênio, além de não proporcionar bom acabamento final.

Ainda na Tabela 1, pode-se observar de maneira geral a eficiência do tratamento químico de sementes em todas as épocas de armazenamento e que, mesmo com a diminuição da germinação das sementes aos 12 meses de armazenamento, houve eficiência do tratamento químico tanto nas sementes revestidas quanto nas não, proporcionando senão igual ou maior porcentagem de germinação em relação às não tratadas.

Pelos resultados da porcentagem de germinação tanto nas sementes tratadas (Figura 2) quanto nas não tratadas (Figura 1), nota-se que do zero aos 3 meses de armazenamento houve um aumento na porcentagem de germinação independente do revestimento e do tratamento utilizado.

Aos 12 meses de armazenamento (Figuras 1 e 2), tanto nas sementes tratadas quimicamente quanto nas não tratadas, nas revestidas ou não, houve um decréscimo na germinação das sementes, podendo ser atribuída à deterioração das mesmas.

$\mathrm{Na}$ Tabela 3 e nas Figuras 3 e 4 estão apresentadas as médias dos resultados do teste de emergência de plântulas de Brachiaria brizantha cv. Marandu de sementes revestidas com diferentes produtos, com e sem tratamento químico ao longo do armazenamento.

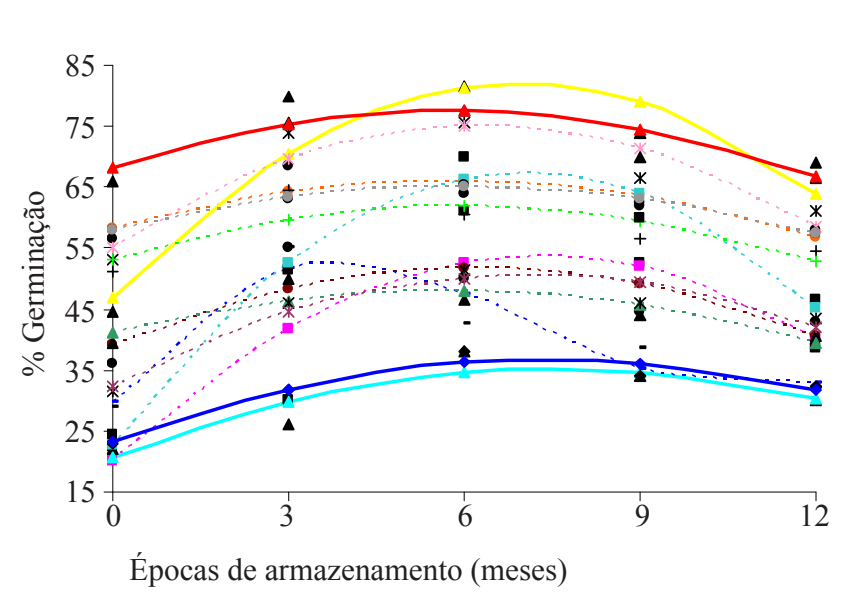

-... Areia + Betonita + PVA, sem TQ (1)

$\ldots$ Areia + Betonita + pol., sem TQ (3)

-... Areia + Calcário + PVA, sem TQ (5)

.... Areia + Calcário + pol., sem TQ (7)

.... Areia +PVA, sem TQ (9)

Areia + Silicato de Cálcio + PVA, sem TQ (11)

Areia + Silicato de Cálcio + pol., sem TQ (13)

- Areia + pol., sem TQ (15)

-. Calcário + Betonita + PVA, sem TQ (17)

-... Calcário + PVA, sem TQ (21)

-... Calcário + Silicato de Cálcio + PVA, sem TQ (23)

-*-. Calcário + Silicato de Cálcio + pol., sem TQ (25)

$\longrightarrow$ Calcário + pol., sem TQ (27)

$\longrightarrow$ Sem revestimento (29)

\section{FIGURA 1. Resultados médios (\%) do teste de germinação de sementes de Brachiaria brizantha cv. Marandu revestidas com diferentes materiais, sem tratamento químico (TQ), aos 0, 3, 6, 9 e 12 meses de armazenamento.}




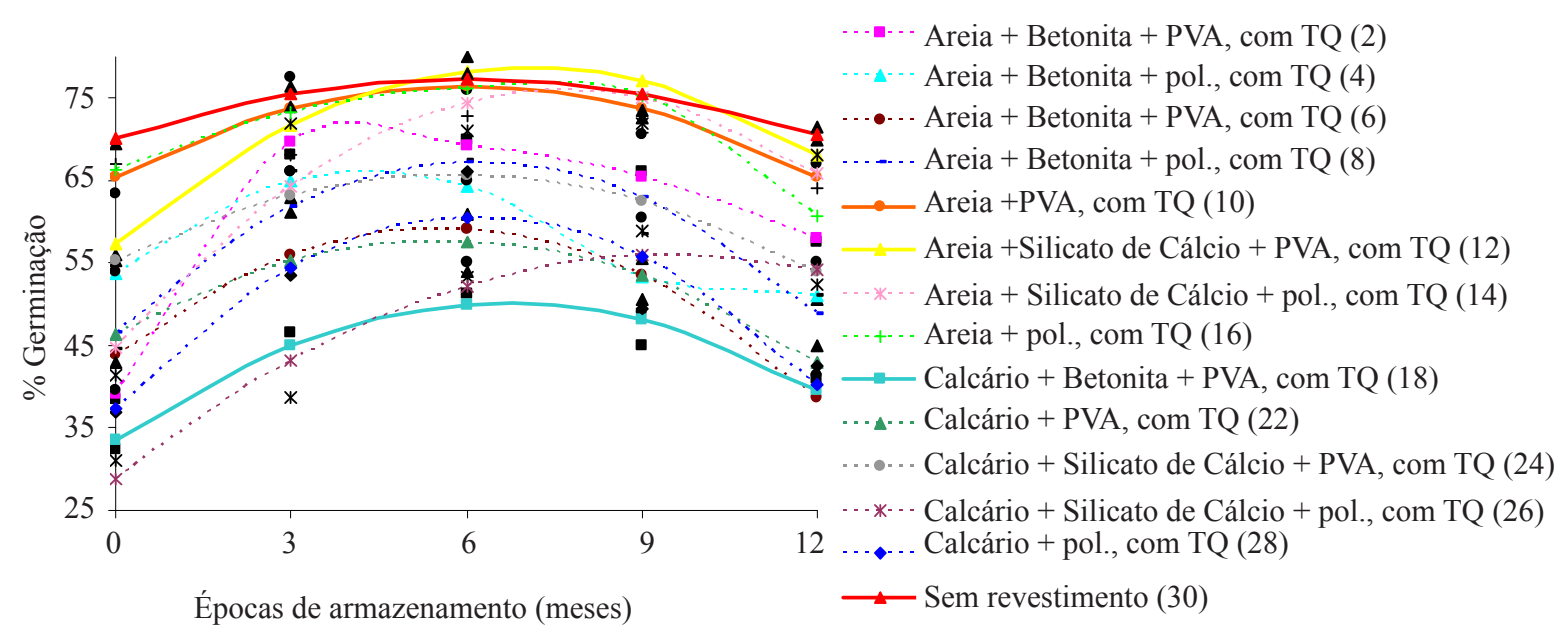

FIGURA 2. Resultados médios (\%) do teste de germinação de sementes de Brachiaria brizantha cv. Marandu revestidas com diferentes materiais, submetidas ao tratamento químico (TQ), aos 0, 3, 6, 9 e 12 meses de armazenamento.

TABELA 3. Resultados médios (\%) do teste de emergência de sementes de Brachiaria brizantha cv. Marandu revestidas com diferentes materiais $\left(\mathrm{A}\right.$ - areia, $\mathrm{C}$ - calcário, $\mathrm{AB}$ - areia + betonita, $\mathrm{CB}$ - calcário + betonita, $\mathrm{ACaSiO}_{3}$ - areia $+\mathrm{CaSiO}_{3}, \mathrm{CCaSiO}_{3}-$ calcário $+\mathrm{CaSiO}_{3}$ ), submetidas ao tratamento químico (TQ) ou não aos 0,3 , 6,9 e 12 meses de armazenamento.

\begin{tabular}{|c|c|c|c|c|c|c|c|c|c|c|}
\hline \multirow{3}{*}{ Revestimento } & \multicolumn{10}{|c|}{ Armazenamento (meses) } \\
\hline & \multicolumn{2}{|c|}{0} & \multicolumn{2}{|c|}{3} & \multicolumn{2}{|c|}{6} & \multicolumn{2}{|c|}{9} & \multicolumn{2}{|c|}{12} \\
\hline & $\begin{array}{c}\text { Com } \\
\text { TQ }\end{array}$ & $\begin{array}{c}\text { Sem } \\
\text { TQ }\end{array}$ & $\begin{array}{c}\text { Com } \\
\text { TQ }\end{array}$ & $\begin{array}{c}\text { Sem } \\
\text { TQ }\end{array}$ & $\begin{array}{c}\text { Com } \\
\text { TQ }\end{array}$ & $\begin{array}{c}\text { Sem } \\
\text { TQ }\end{array}$ & $\begin{array}{c}\text { Com } \\
\text { TQ }\end{array}$ & $\begin{array}{c}\text { Sem } \\
\text { TQ }\end{array}$ & $\begin{array}{c}\text { Com } \\
\text { TQ }\end{array}$ & $\begin{array}{c}\text { Sem } \\
\text { TQ }\end{array}$ \\
\hline$A+P V A$ & $74 \mathrm{Ba}$ & $56 \mathrm{Cb}$ & $61 \mathrm{Ea}$ & $62 \mathrm{Ba}$ & $57 \mathrm{Da}$ & $41 \mathrm{~Eb}$ & $65 \mathrm{Aa}$ & $38 \mathrm{Fb}$ & $55 \mathrm{Ca}$ & $37 \mathrm{Db}$ \\
\hline A + pol. & 79Aa & $69 \mathrm{Ab}$ & $78 \mathrm{Aa}$ & $67 \mathrm{Bb}$ & $76 \mathrm{Aa}$ & $64 \mathrm{Bb}$ & $64 \mathrm{Aa}$ & $62 \mathrm{Ba}$ & $62 \mathrm{Ba}$ & $60 \mathrm{Ba}$ \\
\hline $\mathrm{C}+\mathrm{PVA}$ & $62 \mathrm{Da}$ & $19 \mathrm{~Gb}$ & $58 \mathrm{Ea}$ & $39 \mathrm{Fb}$ & $60 \mathrm{Ca}$ & $43 \mathrm{~Eb}$ & $58 \mathrm{Ba}$ & $45 \mathrm{~Eb}$ & $55 \mathrm{Ca}$ & $30 \mathrm{~Eb}$ \\
\hline $\mathrm{C}+$ pol. & $61 \mathrm{Da}$ & $33 \mathrm{~Eb}$ & $59 \mathrm{Ea}$ & $31 \mathrm{Fb}$ & $52 \mathrm{Da}$ & $29 \mathrm{~Gb}$ & $54 \mathrm{Ca}$ & $24 \mathrm{Hb}$ & $50 \mathrm{Da}$ & $20 \mathrm{Fb}$ \\
\hline $\mathrm{AB}+\mathrm{PVA}$ & $56 \mathrm{Ea}$ & $34 \mathrm{~Eb}$ & $72 \mathrm{Ba}$ & $62 \mathrm{Bb}$ & $52 \mathrm{Da}$ & $45 \mathrm{~Eb}$ & $48 \mathrm{Da}$ & $37 \mathrm{Fb}$ & $48 \mathrm{Da}$ & $33 \mathrm{Db}$ \\
\hline $\mathrm{AB}+$ pol. & $53 \mathrm{Ea}$ & $18 \mathrm{~Gb}$ & $66 \mathrm{Ca}$ & $24 \mathrm{~Gb}$ & $67 \mathrm{Ba}$ & $35 \mathrm{Fb}$ & $55 \mathrm{Ba}$ & $32 \mathrm{~Gb}$ & $62 \mathrm{Ba}$ & $28 \mathrm{~Eb}$ \\
\hline $\mathrm{CB}+\mathrm{PVA}$ & $42 \mathrm{Ga}$ & $16 \mathrm{~Gb}$ & $50 \mathrm{Fa}$ & $54 \mathrm{Da}$ & $62 \mathrm{Ca}$ & $62 \mathrm{Ca}$ & $56 \mathrm{Ba}$ & $52 \mathrm{Db}$ & $54 \mathrm{Ca}$ & $26 \mathrm{~Eb}$ \\
\hline $\mathrm{CB}+$ pol. & $56 \mathrm{Ea}$ & $39 \mathrm{Db}$ & $59 \mathrm{Ea}$ & $47 \mathrm{~Eb}$ & $56 \mathrm{Da}$ & $55 \mathrm{Da}$ & $54 \mathrm{Ca}$ & $53 \mathrm{Da}$ & $52 \mathrm{Da}$ & 49Da \\
\hline $\mathrm{AC}+\mathrm{PVA}$ & $47 \mathrm{Fa}$ & $36 \mathrm{~Eb}$ & $39 \mathrm{Ga}$ & $38 \mathrm{Fa}$ & $39 \mathrm{Fa}$ & $31 \mathrm{~Gb}$ & $38 \mathrm{Ea}$ & $29 \mathrm{~Gb}$ & $36 \mathrm{Ea}$ & $25 \mathrm{~Eb}$ \\
\hline $\mathrm{AC}+$ pol. & $49 \mathrm{Fa}$ & $23 \mathrm{Fb}$ & 64Da & $62 \mathrm{Ba}$ & $55 \mathrm{Da}$ & $44 \mathrm{~Eb}$ & $52 \mathrm{Ca}$ & $40 \mathrm{Fb}$ & $52 \mathrm{Da}$ & $38 \mathrm{Db}$ \\
\hline $\mathrm{ACaSiO}_{3}+\mathrm{PVA}$ & $74 \mathrm{Ba}$ & $67 \mathrm{Bb}$ & $68 \mathrm{Ca}$ & $64 \mathrm{Ba}$ & $67 \mathrm{Bb}$ & $72 \mathrm{Aa}$ & $64 \mathrm{Ab}$ & 71Aa & $61 \mathrm{Bb}$ & 68Aa \\
\hline $\mathrm{ACaSiO}_{3}+$ pol. & $76 \mathrm{Aa}$ & $63 \mathrm{Bb}$ & $73 \mathrm{Ba}$ & $57 \mathrm{Cb}$ & $69 \mathrm{Ba}$ & $52 \mathrm{Db}$ & $68 \mathrm{Aa}$ & $50 \mathrm{Db}$ & 68Aa & $48 \mathrm{Cb}$ \\
\hline $\mathrm{CCaSiO}_{3}+\mathrm{PVA}$ & $72 \mathrm{Ba}$ & $65 \mathrm{Bb}$ & $63 \mathrm{Da}$ & $59 \mathrm{Cb}$ & $62 \mathrm{Ca}$ & $59 \mathrm{Ca}$ & $58 \mathrm{Ba}$ & $57 \mathrm{Ca}$ & $54 \mathrm{Ca}$ & $54 \mathrm{Ca}$ \\
\hline $\mathrm{CCaSiO}_{3}+$ pol. & $68 \mathrm{Ca}$ & $54 \mathrm{Cb}$ & $61 \mathrm{Ea}$ & $53 \mathrm{Db}$ & $45 \mathrm{~Eb}$ & $56 \mathrm{Da}$ & $29 \mathrm{Fb}$ & $54 \mathrm{Ca}$ & $25 \mathrm{Fb}$ & $52 \mathrm{Ca}$ \\
\hline $\mathrm{S} /$ revestimento & 79Aa & $72 \mathrm{Aa}$ & 76Aa & 71Aa & $68 \mathrm{Ba}$ & $72 \mathrm{Aa}$ & $65 \mathrm{Aa}$ & 69Aa & $64 \mathrm{Ba}$ & $65 \mathrm{Aa}$ \\
\hline
\end{tabular}

Médias seguidas pela mesma letra maiúscula na coluna e minúscula na linha para cada época de armazenamento não diferem estatisticamente entre si pelo Teste de Scott-Knott, a 5\% de probabilidade. 


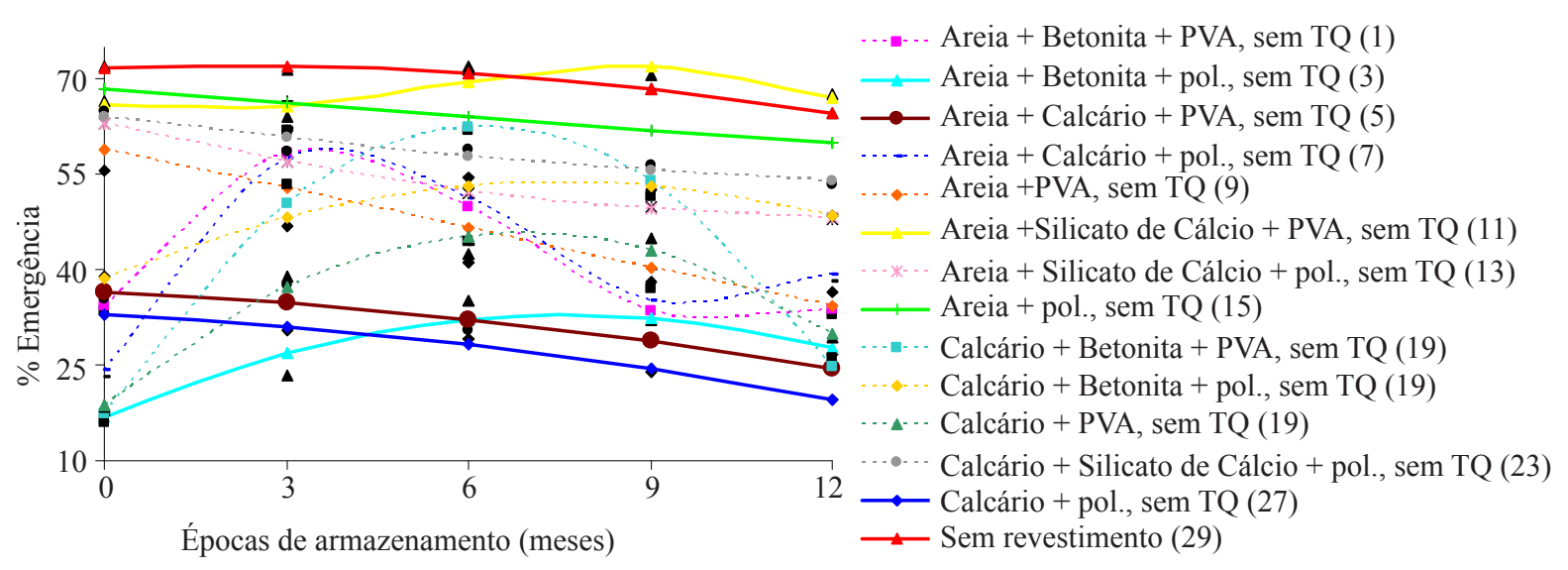

FIGURA 3 Resultados médios (\%) do teste de emergência de sementes de Brachiaria brizantha cv. Marandu revestidas com diferentes materiais, sem tratamento químico (TQ) aos 0, 3, 6, 9 e 12 meses de armazenamento.

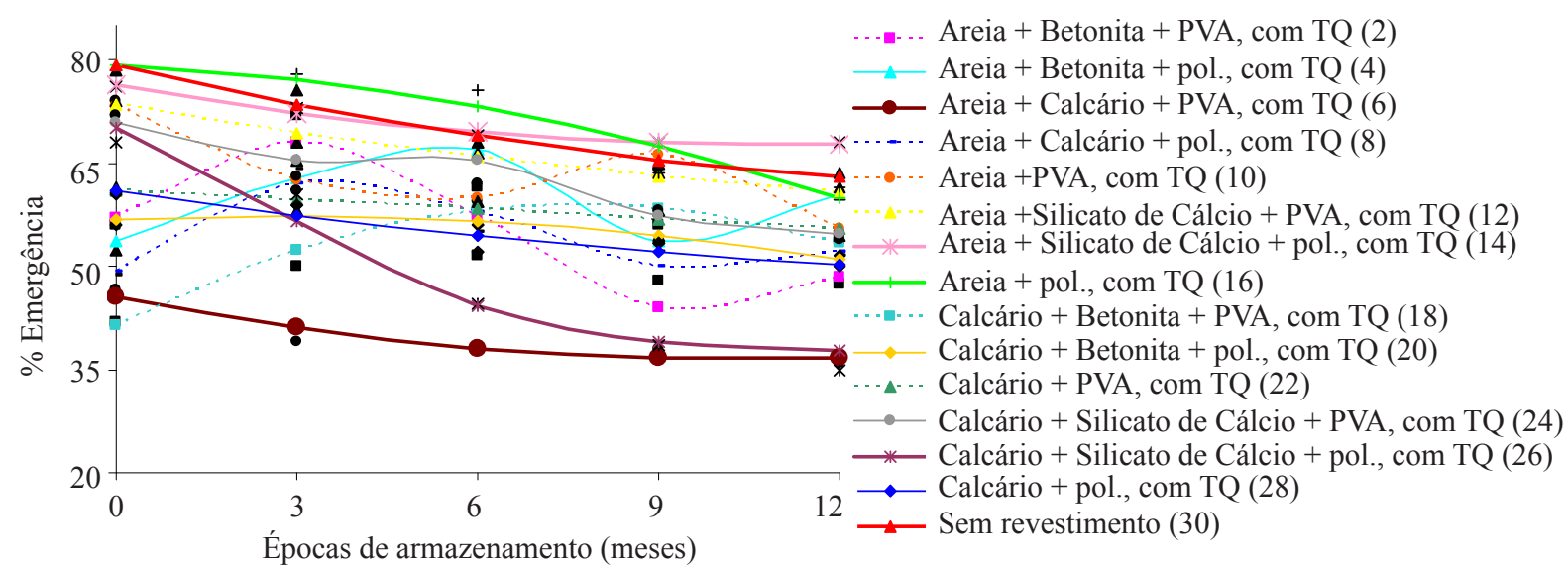

FIGURA 4. Resultados médios (\%) do teste de emergência de sementes de Brachiaria brizantha cv. Marandu revestidas com diferentes materiais, submetidas ao tratamento químico (TQ), aos 0, 3, 6, 9 e 12 meses de armazenamento.

Verifica-se que nas sementes recém-armazenadas (zero mês de armazenamento), tratadas quimicamente, as revestidas com Areia + polímero e Areia $+\mathrm{CaSiO}_{3}+$ polímero resultaram em maiores porcentagens de germinação, não diferindo das sementes sem revestimento. Menor porcentagem de emergência foi obtida com a utilização das sementes revestidas com Calcário + Betonita + PVA. Aos três meses de armazenamento somente o tratamento com Areia + polímero não diferiram do tratamento testemunha, e a menor porcentagem foi constatada nas sementes revestidas com Areia + Calcário + PVA. Já aos seis meses de armazenamento as sementes revestidas com Areia + polímero, apresentaram emergência de plântulas superior à testemunha e aos demais revestimentos, e o menor resultado foi com a utilização das sementes revestidas com Areia + Calcário + PVA. Aos nove meses de armazenamento, nas sementes revestidas com Areia
+ PVA, Areia + polímero, Areia $+\mathrm{CaSiO}_{3}+$ PVA e Areia + $\mathrm{CaSiO}_{3}+$ polímero foram observados os maiores valores de germinação, não diferindo significativamente da testemunha (sem revestimento). Menores valores foram observados nas sementes revestidas com Calcário $+\mathrm{CaSiO}_{3}+$ polímero. Aos 12 meses de armazenamento, Areia $+\mathrm{CaSiO}_{3}+$ polímero superou a testemunha e os demais revestimentos e as sementes revestidas com Calcário $+\mathrm{CaSiO}_{3}+$ polímero resultou no pior resultado.

Os revestimentos que contém silicato de cálcio $\left(\mathrm{CaSiO}_{3}\right)$ a partir dos nove meses de armazenamento proporcionaram bons resultados tanto para as sementes tratadas quimicamente quanto para as não tratadas. Segundo Reis et al. (2007), diversos pesquisadores relatam que a atuação do silício nos solos e a sua reposição por meio de adubação podem exercer efeito benéfico na agricultura, 
conferindo maior tolerância da cultura ao déficit hídrico, maior resistência ao ataque de pragas e doenças, melhora na arquitetura da planta e, consequentemente, sua capacidade fotossintética, gerando incremento na produção das culturas. Portanto, pode-se inferir que o Si utilizado no revestimento tenha promovido algum efeito fungitóxico aos fungos de armazenamento o que favoreceu a emergência das plântulas de Brachiaria brizantha cv. Marandu.

Ainda na Tabela 3, nos resultados do teste de emergência para as sementes sem tratamento químico foram observados, nas recém-armazenadas, maior porcentagem nas sementes revestidas com Areia + polímero que as demais, não diferindo estatisticamente da testemunha. Menores valores foram observados naquelas revestidas com Calcário + PVA, Areia + Betonita + polímero e Calcário + Betonita + PVA; aos três meses de armazenamento nenhum revestimento foi superior à testemunha e o menor valor foi observado nas sementes revestidas com Areia + Betonita + polímero. Aos seis meses verificou-se maior porcentagem nas sementes revestidas com Areia $+\mathrm{CaSiO}_{3}+\mathrm{PVA}$, não apresentando diferença em relação à testemunha, e menores nas sementes revestidas com Calcário + polímero e Areia + Calcário + PVA. Com nove meses de armazenamento das sementes revestidas com Areia $+\mathrm{CaSiO}_{3}+$ polímero obteve-se maior porcentagem de emergência, não diferenciando da testemunha. Menor porcentagem de emergência foi observada naquelas revestidas com Calcário + polímero. Enfim aos 12 meses, as sementes revestidas com Areia $+\mathrm{CaSiO}_{3}+\mathrm{PVA}$ foi superior aos demais não diferindo da testemunha e as revestidas com Calcário + polímero obteve-se menores valores de emergência.

Observa-se, de uma maneira geral, que as sementes revestidas com Areia + polímero, Areia $+\mathrm{CaSiO}_{3}+\mathrm{PVA} \mathrm{e}$ Areia $+\mathrm{CaSiO}_{3}+$ polímero foram superiores em relação às revestidas com as demais combinações de materiais no teste de emergência tanto as tratadas quanto as não tratadas.

Os índices de velocidade de emergência estão apresentados na Tabela 5 e Figuras 5 e 6 . Pode-se verificar que ao zero (recém-armazenadas) e aos três meses de armazenamento, nas sementes sem e com tratamento químico, nenhum revestimento superou a testemunha. Já aos seis, nove e 12 meses, sementes com tratamento químico e revestidas com Areia + polímero foram superiores às demais, não apresentando diferença significativa em relação à testemunha e aos seis, nove e 12 meses, sem tratamento químico, as sementes revestidas com Areia $+\mathrm{CaSiO}_{3}+\mathrm{PVA}$ apresentaram maior índice não diferindo da testemunha.

TABELA 5. Resultados médios do índice de velocidade de emergência de sementes de Brachiaria brizantha cv. Marandu revestidas com diferentes materiais $(\mathrm{A}$ - areia, $\mathrm{C}$ - calcário, $\mathrm{AB}$ - areia + betonita, $\mathrm{CB}$ - calcário + betonita, $\mathrm{ACaSiO}_{3}-$ areia $+\mathrm{CaSiO}_{3}, \mathrm{CCaSiO}_{3}-$ calcário $+\mathrm{CaSiO}_{3}$ ), submetidas ao tratamento químico (TQ) ou não aos 0, 3, 6, 9 e 12 meses de armazenamento.

\begin{tabular}{|c|c|c|c|c|c|c|c|c|c|c|}
\hline \multirow{3}{*}{ Revestimento } & \multicolumn{10}{|c|}{ Armazenamento (meses) } \\
\hline & \multicolumn{2}{|c|}{0} & \multicolumn{2}{|c|}{3} & \multicolumn{2}{|c|}{6} & \multicolumn{2}{|c|}{9} & \multicolumn{2}{|c|}{12} \\
\hline & $\begin{array}{c}\text { Com } \\
\text { TQ }\end{array}$ & $\begin{array}{c}\text { Sem } \\
\text { TQ }\end{array}$ & $\begin{array}{c}\text { Com } \\
\text { TQ }\end{array}$ & $\begin{array}{c}\text { Sem } \\
\text { TQ }\end{array}$ & $\begin{array}{c}\text { Com } \\
\text { TQ }\end{array}$ & $\begin{array}{c}\text { Sem } \\
\text { TQ }\end{array}$ & $\begin{array}{c}\text { Com } \\
\text { TQ }\end{array}$ & $\begin{array}{c}\text { Sem } \\
\text { TQ }\end{array}$ & $\begin{array}{c}\text { Com } \\
\text { TQ }\end{array}$ & $\begin{array}{l}\text { Sem } \\
\text { TQ }\end{array}$ \\
\hline$A+P V A$ & $7,54 \mathrm{Ba}$ & $6,69 \mathrm{Bb}$ & $6,60 \mathrm{Ba}$ & $4,36 \mathrm{Db}$ & $5,36 \mathrm{Ba}$ & $3,06 \mathrm{Cb}$ & $5,23 \mathrm{Ba}$ & $2,96 \mathrm{Cb}$ & $4,97 \mathrm{Ba}$ & $2,95 \mathrm{Cb}$ \\
\hline $\mathrm{A}+$ pol. & $8,05 \mathrm{Ba}$ & $6,51 \mathrm{Bb}$ & $6,91 \mathrm{Ba}$ & $4,93 \mathrm{Cb}$ & 6,71Aa & $4,77 \mathrm{Bb}$ & $6,41 \mathrm{Aa}$ & $4,34 \mathrm{Bb}$ & $6,03 \mathrm{Aa}$ & $4,04 \mathrm{Bb}$ \\
\hline $\mathrm{C}+\mathrm{PVA}$ & $5,00 \mathrm{Ea}$ & $1,92 \mathrm{Fb}$ & $5,14 \mathrm{Ca}$ & $2,62 \mathrm{~Eb}$ & $5,06 \mathrm{Ca}$ & $3,19 \mathrm{Cb}$ & $4,96 \mathrm{Ba}$ & $3,54 \mathrm{Cb}$ & $4,64 \mathrm{Ca}$ & $2,52 \mathrm{Db}$ \\
\hline $\mathrm{C}+$ pol. & $6,25 \mathrm{Da}$ & $2,69 \mathrm{Fb}$ & $4,28 \mathrm{Da}$ & $2,57 \mathrm{~Eb}$ & $3,93 \mathrm{Da}$ & $2,13 \mathrm{Db}$ & $3,51 \mathrm{Da}$ & $1,96 \mathrm{Db}$ & $3,90 \mathrm{Ca}$ & $1,28 \mathrm{~Eb}$ \\
\hline $\mathrm{AB}+\mathrm{PVA}$ & $3,80 \mathrm{Fa}$ & $2,37 \mathrm{Fb}$ & $5,00 \mathrm{Ca}$ & $5,28 \mathrm{Cb}$ & $4,52 \mathrm{Ca}$ & $3,90 \mathrm{Bb}$ & $3,71 \mathrm{Da}$ & $3,16 \mathrm{Ca}$ & $3,27 \mathrm{Da}$ & $2,99 \mathrm{Ca}$ \\
\hline $\mathrm{AB}+$ pol. & $4,21 \mathrm{Fa}$ & $0,99 \mathrm{Hb}$ & $6,82 \mathrm{Ba}$ & $1,25 \mathrm{Fb}$ & $4,82 \mathrm{Ca}$ & $2,28 \mathrm{Db}$ & $4,46 \mathrm{Ca}$ & $2,16 \mathrm{Db}$ & $4,30 \mathrm{Ca}$ & $2,00 \mathrm{~Eb}$ \\
\hline $\mathrm{CB}+\mathrm{PVA}$ & $5,00 \mathrm{Ea}$ & $2,30 \mathrm{Fb}$ & $5,33 \mathrm{Ca}$ & $3,97 \mathrm{Db}$ & $4,79 \mathrm{Ca}$ & $4,44 \mathrm{Ba}$ & $4,49 \mathrm{Ca}$ & $3,52 \mathrm{Cb}$ & $4,33 \mathrm{Ca}$ & $2,46 \mathrm{Db}$ \\
\hline $\mathrm{CB}+$ pol. & $6,40 \mathrm{Da}$ & $3,95 \mathrm{~Eb}$ & $4,84 \mathrm{Ca}$ & $4,80 \mathrm{Ca}$ & $4,49 \mathrm{Ca}$ & $4,49 \mathrm{Ba}$ & $4,31 \mathrm{Ca}$ & $4,42 \mathrm{Ba}$ & $4,10 \mathrm{Ca}$ & $4,11 \mathrm{Ba}$ \\
\hline $\mathrm{AC}+\mathrm{PVA}$ & $4,63 \mathrm{Ea}$ & $4,12 \mathrm{Ea}$ & $3,29 \mathrm{Ea}$ & $2,82 \mathrm{Ea}$ & $3,38 \mathrm{Da}$ & $2,32 \mathrm{Db}$ & $3,35 \mathrm{Da}$ & $2,12 \mathrm{Db}$ & $2,97 \mathrm{Da}$ & $2,05 \mathrm{~Eb}$ \\
\hline $\mathrm{AC}+$ pol. & $4,13 \mathrm{Fa}$ & $1,65 \mathrm{~Gb}$ & $5,46 \mathrm{Ca}$ & $5,06 \mathrm{Ca}$ & $4,63 \mathrm{Ca}$ & $3,70 \mathrm{Cb}$ & $4,53 \mathrm{Ca}$ & $3,32 \mathrm{Cb}$ & $4,27 \mathrm{Ca}$ & $2,44 \mathrm{Db}$ \\
\hline $\mathrm{ACaSiO}_{3}+\mathrm{PVA}$ & $7,62 \mathrm{Ba}$ & $6,86 \mathrm{Bb}$ & $6,16 \mathrm{Ba}$ & $5,94 \mathrm{Ba}$ & $5,81 \mathrm{Ba}$ & 5,61Aa & $5,62 \mathrm{Ba}$ & $5,54 \mathrm{Aa}$ & $5,36 \mathrm{Ba}$ & $5,02 \mathrm{Aa}$ \\
\hline $\mathrm{ACaSiO}_{3}+$ pol. & 7,32Ca & $5,62 \mathrm{Cb}$ & $6,47 \mathrm{Ba}$ & $4,83 \mathrm{Cb}$ & $5,49 \mathrm{Ba}$ & $3,38 \mathrm{Cb}$ & $5,25 \mathrm{Ba}$ & $3,33 \mathrm{Cb}$ & $5,30 \mathrm{Ba}$ & $3,13 \mathrm{Cb}$ \\
\hline $\mathrm{CCaSiO}_{3}+\mathrm{PVA}$ & 7,19Ca & $6,57 \mathrm{Bb}$ & $5,22 \mathrm{Ca}$ & $5,25 \mathrm{Ca}$ & $4,68 \mathrm{Ca}$ & $4,24 \mathrm{Ba}$ & $4,48 \mathrm{Ca}$ & $4,05 \mathrm{Ba}$ & $4,17 \mathrm{Ca}$ & $3,78 \mathrm{Ba}$ \\
\hline $\mathrm{CCaSiO}_{3}+$ pol. & $6,83 \mathrm{Ca}$ & $4,88 \mathrm{Db}$ & $4,49 \mathrm{Da}$ & $4,79 \mathrm{Ca}$ & $3,33 \mathrm{Db}$ & $4,25 \mathrm{Ba}$ & $2,14 \mathrm{~Eb}$ & $4,36 \mathrm{Ba}$ & $2,01 \mathrm{~Eb}$ & $3,93 \mathrm{Ba}$ \\
\hline $\mathrm{S} /$ revestimento & $8,85 \mathrm{Aa}$ & $9,167 \mathrm{Aa}$ & 7,74Aa & $8,00 \mathrm{Aa}$ & $6,77 \mathrm{Aa}$ & $6,10 \mathrm{Ab}$ & $6,75 \mathrm{Aa}$ & $5,75 \mathrm{Ab}$ & $6,54 \mathrm{Aa}$ & $5,55 \mathrm{Ab}$ \\
\hline
\end{tabular}

Médias seguidas pela mesma letra maiúscula na coluna e minúscula na linha para cada época de armazenamento não diferem estatisticamente entre si pelo Teste de Scott-Knott, a 5\% de probabilidade. 


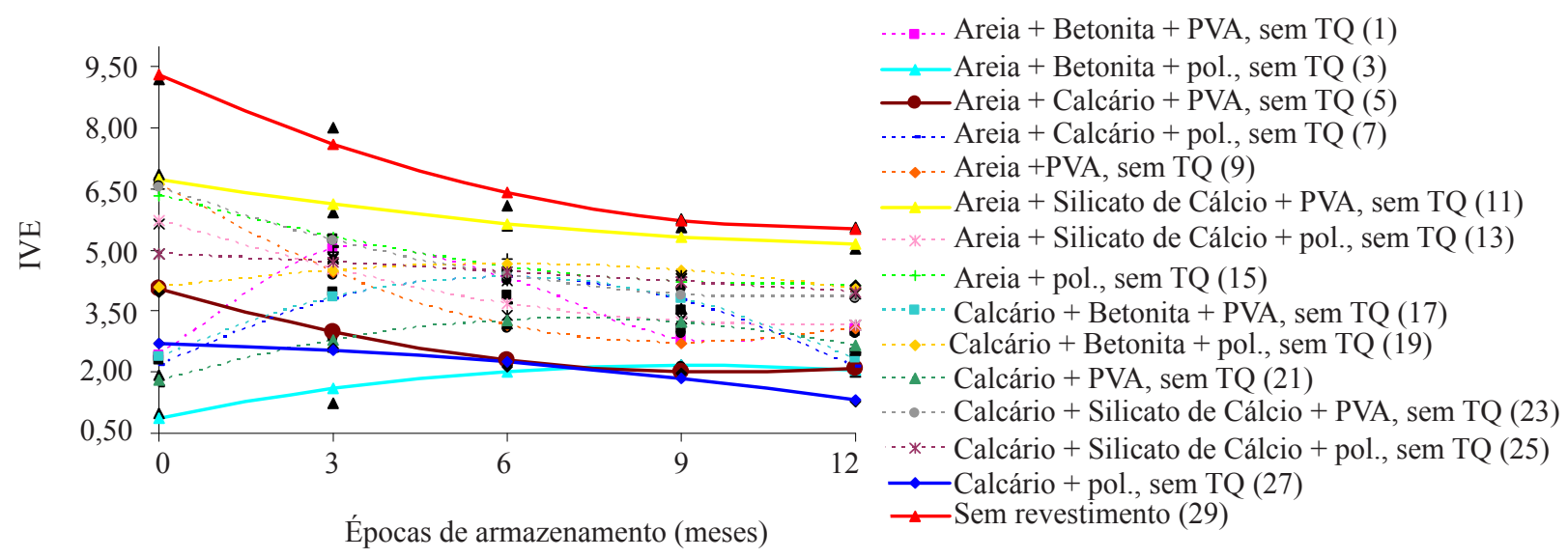

FIGURA 5. Resultados médios do índice de velocidade de emergência de sementes de Brachiaria brizantha cv. Marandu revestidas com diferentes materiais, sem tratamento químico (TQ), aos 0, 3, 6, 9 e 12 meses de armazenamento.

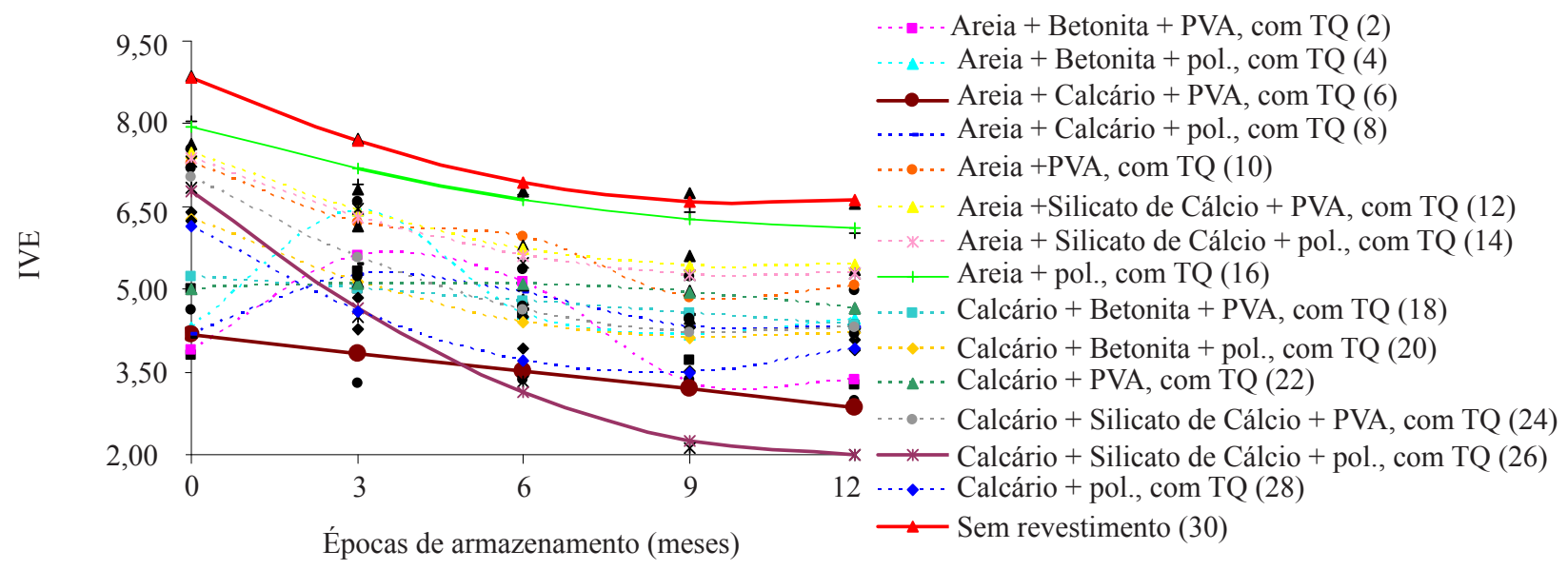

FIGURA 6. Resultados médios do índice de velocidade de emergência de sementes de Brachiaria brizantha cv. Marandu revestidas com diferentes materiais, submetidas ao tratamento químico (TQ), aos 0, 3, 6, 9 e 12 meses de armazenamento.

Os menores índices de velocidade de emergência para as sementes com tratamento químico (Tabela 5) foram observados nas sementes revestidas com Areia + Betonita + PVA e Areia + Betonita + polímero e Areia + Calcário + polímero nas sementes recém-armazenadas; Areia + Calcário + PVA aos três meses de armazenamento; Calcário + polímero, Areia + Calcário + PVA e Calcário $+\mathrm{CaSiO}_{3}$ + polímero aos seis meses de armazenamento; Calcário + $\mathrm{CaSiO}_{3}+$ polímero aos nove meses de armazenamento e Areia + Betonita + PVA e Areia + Calcário + PVA aos
12 meses. Já para as sementes sem tratamento químico foram: Areia + Betonita + polímero nas sementes recém armazenadas e aos três meses de armazenamento; Calcário + polímero, Areia + Betonita + polímero e Areia + Calcário + PVA aos 6 meses de armazenamento; Calcário + polímero, Areia + Betonita + polímero e Areia + Calcário + PVA aos nove meses de armazenamento e por fim Calcário + polímero e Calcário $+\mathrm{CaSiO}_{3}+$ polímero aos 12 meses.

Dessa forma, verifica-se na Tabela 5 , que o revestimento provoca a redução do índice de velocidade de emergência 
em todas as épocas de armazenamento, nas sementes tratadas quimicamente ou não. Já nas sementes revestidas com Areia + Betonita + polímero pôde-se observar os menores índices de velocidade de emergência de plântulas em todas as épocas de armazenamento, para as sementes não tratadas. O maior índice de velocidade de emergência das sementes sem revestimento já era de se esperar, pois o revestimento constitui uma barreira física para a protrusão radicular, retardando assim sua germinação.

Como no teste de emergência, os valores do índice de emergência diminuíram com o tempo de armazenamento (Figuras 4 e 5). Alguns fatores podem estar relacionados a esta redução, como o avanço do processo natural de deterioração e aumento da colonização das sementes por fungos. Sabe-se que sementes em avançado processo de deterioração, podem germinar com velocidade mais baixa.

\section{CONCLUSÕES}

- Sementes revestidas com Areia + PVA e Areia + $\mathrm{CaSiO}_{3}+$ PVA ou polímero, tratadas quimicamente, mantém sua qualidade fisiológica ao longo dos 12 meses de armazenamento;

- O tratamento químico com fungicida Carbendazim + Thiram e inseticida Fipronil nas sementes revestidas ou não, é eficiente no controle de fungos e promove melhor desempenho fisiológico ao longo do armazenamento;

- O revestimento com Betonita e/ou Calcário prejudica a qualidade fisiológica das sementes de Brachiaria brizantha cv. Marandu armazenadas por um período de 12 meses;

- O revestimento de sementes promove redução da velocidade de germinação e emergência de plântulas de Brachiaria brizantha cv. Marandu.

\section{REFERÊNCIAS}

BONOME, L.T.S. Condicionamento fisiológico e revestimento de sementes de Brachiaria brizantha cultivar Marandu. 2003. 99f. Dissertação (Mestrado em Agronomia)-Universidade Federal de Lavras, Lavras.

BRASIL. Ministério da Agricultura e Reforma Agrária. Secretaria Nacional de Defesa Agropecuária. Departamento
Nacional de Defesa Vegetal. Coordenação de Laboratório Vegetal. Regras para análise de sementes. Brasília, DF, 1992. 365p.

FERREIRA, D.F. Análises estatísticas por meio do SISVAR para Windows versão 4.0. In: REUNIÃO ANUAL DA REGIÃO BRASILEIRA DA SOCIEDADE INTERNACIONAL DE BIOMETRIA, 45., 2000, São Carlos. Programas e Resumos... São Carlos: UFSCAR, 2000. p.235.

GOULART, A.C.P. Influência do grafite adicionado às sementes de soja e algodão na eficiência do tratamento com fungicidas. Dourados: Embrapa Agropecuária Oeste, 2000. 27p. (Embrapa Agropecuária Oeste. Boletim de Pesquisa, 8).

MAGUIRE, J.D. Spped og germination aid in selection and evaluation for seedling and vigour. Crop Science, v.2, n.2, p.176-177, Mar./Apr. 1962.

MARTINS, L.; LAGO, A.A.; GROTH, D. Valor cultural de sementes de Brachiaria brizantha (Hochst. Ex A. Rich) Stapf durante o armazenamento. Revista Brasileira de Sementes, v.20, n.1, p.60-64, fev. 1998.

OLIVEIRA, J.A.; CARVALHO, M.L.M. de; VIEIRA, M. das G.G.C.; PINHO, E.V.R. von. Comportamento de sementes de milho colhidas por diferentes métodos, sob condições de armazém convencional. Ciência e Agrotecnologia, v.23, n.2, p.289-302, mar./abr. 1999.

PEREIRA, C.E.; OLIVEIRA, J.A.; GUIMARÃES, R.M.; VIEIRA, A.R.; SILVA, J B. C. da. Condicionamento fisiológico e revestimento de sementes de pimentão. Revista Ciência Agronômica, v.36, n.1, p.74-81, jan./abr. 2005.

REIS, T.H.P.; GUIMARÃES, P.T.G.; FIGUEIREDO, F.C.; POZZA, A.A.A.; NOGUEIRA, F.D.; RODRIGUES, C.R. O silício na nutrição e defesa de plantas. Belo Horizonte: EPAMIG, 2007. 120p. (EPAMIG. Boletim Técnico, 82).

SILVA, J.B.C.; SANTOS, P.E.C.; NASCIMENTO, W.M. Desempenho de sementes peletizadas de alface em função do material cimentante e da temperatura de secagem dos péletes. Horticultura Brasileira, v.20, n.1, p.67-70, fev. 2002. 